\title{
Seawater-drowning-induced acute lung injury: From molecular mechanisms to potential treatments (Review)
}

\author{
FAGUANG JIN and CONGCONG LI
}

\begin{abstract}
Department of Respiratory and Critical Care Medicine, Tangdu Hospital, The Fourth Military Medical University, Xi'an, Shaanxi 710038, P.R. China
\end{abstract}

Received December 10, 2015; Accepted January 26, 2017

DOI: $10.3892 /$ etm.2017.4302

\begin{abstract}
Drowning is a crucial public safety problem and is the third leading cause of accidental fatality, claiming $\sim 372,000$ lives annually, worldwide. In near-drowning patients, acute lung injury (ALI) or acute respiratory distress syndrome (ARDS) is one of the most common complications. Approximately $1 / 3$ of near-drowning patients fulfill the criteria for ALI or ARDS. In the present article, the current literature of near-drowning, pathophysiologic changes and the molecular mechanisms of seawater-drowning-induced ALI and ARDS was reviewed. Seawater is three times more hyperosmolar than plasma, and following inhalation of seawater the hyperosmotic seawater may cause serious injury in the lung and alveoli. The perturbing effects of seawater may be primarily categorized into insufficiency of pulmonary surfactant, blood-air barrier disruption, formation of pulmonary edema, inflammation, oxidative stress, autophagy, apoptosis and various other hypertonic stimulation. Potential treatments for seawater-induced ALI/ARDS were also presented, in addition to suggestions for further studies. A total of nine therapeutic strategies had been tested and all had focused on modulating the over-activated immunoreactions. In conclusion, seawater drowning is a complex injury process and the exact mechanisms and potential treatments require further exploration.
\end{abstract}

\section{Contents}

1. Introduction

2. Pathophysiology of seawater-drowning induced acute lung injury

3. Perturbing effects of seawater

Correspondence to: Dr Faguang Jin, Department of Respiratory and Critical Care Medicine, Tangdu Hospital, The Fourth Military Medical University, 1 Xinsi Road, Xi'an, Shaanxi 710038, P.R. China E-mail: jinfag@fmmu.edu.cn

Key words: acute respiratory distress syndrome, acute lung injury, near-drowning, seawater
4. Potential treatments and therapeutic targets of seawater-drowning induced acute lung injury

5. Summary and conclusions

\section{Introduction}

Drowning is the third leading cause of accidental fatality (1) and claims $\sim 372,000$ lives annually, worldwide $(2,3)$. Over $50 \%$ of the drowning victims that result in fatality are $<25$ years-old $(1,2)$; however, this serious health threat is often neglected. In near-drowning patients, lung injury is one of the most common complications (4,5). Furthermore, $\sim 1 / 3$ of near-drowning patients fulfill the criteria for acute lung injury (ALI) or acute respiratory distress syndrome (ARDS) (4). ALI/ARDS has been acknowledged as a common and lethal disease since it was first described in 1967 (6). It is a life-threatening disorder with a mortality rate ranging from $25-40 \%$ and so cannot be ignored (6). Pneumonia, aspiration, shock and severe sepsis are the primary triggers (7). Furthermore, a result of this complication is the infiltration of neutrophils into the alveolar space, the release pro-inflammatory cytokines, which causes leakage of edema fluid and mismatch of ventilation and perfusion $(6,7)$. In recent years, taking vacations close to the sea or exploiting marine resources has becoming more popular and with this the frequency of seawater drowning accidents have correspondingly increased. Therefore, it is essential that the mechanisms of seawater-drowning-induced ALI be fully elucidated.

\section{Pathophysiology of seawater-drowning-induced acute lung injury}

Physiologic changes of water drowning. As stated by Layon and Modell (1), a detrimental consequence of drowning is primary respiratory impairment from submersion/immersion in a liquid medium. When suddenly immersed in water, victims will hold their breath, which results in oxygen depletion and carbon dioxide accumulation, a condition that is not sustainable. Subsequently, victims eventually become hypercarbic and hypoxemic; thus, in response, victims may begin to breathe, subsequently inhaling water. This further impairs the victims' ability to breathe and exchange air normally $(1,3,8)$. Drowning or near-drowning prevents victims from breathing, which subsequently induces hypoxia. It is widely acknowledged 
that hypoxia provokes multiple complications, such as cerebral hypoxia and cardiovascular disorders (5); however, hypoxia has also been associated with initiating ALI/ARDS $(9,10)$.

Pathophysiological changes in the lung after seawater inhalation. Seawater is a hyperosmotic liquid that is low in temperature and contains a high content of sodium, calcium and substantial quantities of bacteria (11). Additionally, seawater is three times more hyperosmolar than plasma (942 vs. $300 \mathrm{mOsm} / \mathrm{kg}$, respectively) $(11,12)$. Following inhalation of seawater, the hyperosmotic and cold seawater may produce a strong injury stimulus (such as inflammation, DNA damage and apoptosis) in the lung and alveoli (13-15). As the osmotic pressure of seawater is higher than that of plasma, the fluid in the surrounding tissue space and pulmonary capillaries enters the alveolar space, resulting in pulmonary edema and hypoxia (16-18), which may result in ALI/ARDS.

The presence of water in the alveoli and airways obstructs ventilation and blood-gas exchange, triggering immediate hypoxia (19). As reported in multiple studies $(12,18,19)$, the severity of seawater-induced pulmonary edema is 3 -fold higher when compared with edema caused by freshwater. Pathological alterations occur when lungs are perfused with seawater, including alveolar septum widening, alveolar collapse and alveolar-capillary membrane damage $(15,20)$. Folkesson et al (12) indicated that instilling a hyperosmotic liquid, such as seawater, into the trachea of rabbits may increase alveolar-capillary membrane permeability, resulting in the exudation of water, ions and proteins, as well as neutrophils and macrophages (20). As a consequence, massive inflammatory mediators are released and elicit further damage to lung tissue and cells.

\section{Perturbing effects of seawater}

Seawater drowning may cause ALI/ARDS; however, the underlying molecular mechanisms have not yet been clearly elucidated. The present review has a predominant focus on the molecular networks affected by seawater and the resulting cellular alterations. Seawater-induced changes are summarized in Table I (13-17,19-32) and are elucidated in detail.

Insufficiency of pulmonary surfactant. Pulmonary surfactant is a lipid-protein complex synthetized and secreted by type II alveolar epithelial cells (33). It is comprised of four types of protein: Surfactant protein (SP)-A, SP-B, SP-C and SP-D (34). Pulmonary surfactant secretion aids in the reduction of alveolar surface tension, maintains alveolar opening and prevents lung interstitial proteins and fluids leaking into the alveolar cavity (35). Multiple studies have proposed that the content of pulmonary surfactant may be an important severity indicator in critically ill patients with lung diseases $(36,37)$. Seawater aspiration and interstitial fluid exudation dilute and wash out pulmonary surfactant $(1,3,30)$. Inhalation of seawater may cause lung inflammation and type II alveolar epithelial cell damage. This damage may result from perturbing the physiological function of epithelial cells and inhibiting surfactant synthesis and secretion (30). Alternatively, a deficiency of pulmonary surfactants subsequently triggers a decrease in lung compliance, which increases the risk of alveolar collapse, in addition to increasing cell permeability to fluid. Furthermore, this event provokes alterations in lung ventilation/perfusion ratios $(38,39)$, which ultimately may result in ALI/ARDS.

Blood-air barrier disruption. Gas exchange between alveoli and the blood in surrounding capillaries is dependent on the lung blood-air barrier (40). Various causes of lung diseases may inflict damage on lung blood-air barrier function, thereby affecting the respiratory function of the lungs $(41,42)$. However, barrier disruption differs with injury type. Pulmonary edema causes pulmonary interstitium thickening and alters the gas exchange process by increasing the distance for gas exchange to occur (43), whereas lipopolysaccharide-induced acute lung injury predominantly damages pulmonary microvascular endothelial cells and destroys their barrier function, thereby causing inflammatory cell aggregation, adhesion, exudation and secretion of various inflammatory cytokines and chemokines (44). As a result, the blood barrier is further damaged.

In seawater-induced lung injury, the components of seawater, including bacteria and viruses, affect the regulation of pulmonary surfactant and the alveolar epithelium directly. Alteration of the pulmonary surfactant layer may generate pulmonary surfactant deficiency (30) and may also affect the composition of alveoli by promoting alveolar epithelial cell damage and apoptosis and subsequently resulting in barrier function alteration (13). The hypertonic nature of seawater elicits direct epithelial cell stimulation and causes the cells to contract (31), increasing the gap between cells and therefore increasing the permeability of these cells (12). The RhoA/Rho kinase pathway participates in the cytoskeletal contractile response (45). Rho-associated coiled-coil forming protein kinase (ROCK) promotes phosphorylation of the light-chain of myosin (MLC) though MLC phosphatase (46). Multiple studies $(45,47)$ have demonstrated that the RhoA/ROCK pathway regulates cell contraction and modulates the actin cytoskeleton. Seawater has been indicated to induce the RhoA/Rho kinase pathway and promotes the phosphorylation of myosin phosphatase target subunit 1 , both in vivo and in vitro (31).

Previous reports have revealed that seawater inhalation damages tight junctions and gap junctions between cells, thus affecting alveolar cell permeability, function and communication, ultimately promoting lung edema formation $(16,26)$. Connexin 43 is located in gap junction channels and connects the cytoplasm between adjacent cells (48). Furthermore, connexin 43 is able to rapidly exchange ions and intracellular signaling molecules. Notably, in the presence of seawater, connexin 43 has been indicated to upregulate the phosphorylation of Ser368, while p-connexin 43 downregulation protects the barrier function and palliates lung edema $(16,26)$.

Formation of pulmonary edema. Pulmonary edema formation is an additional critical mechanism associated with ALI and ARDS (29). Following inhalation of seawater, water flows directly into alveoli and the osmotic pressure gradient promotes water retention within alveoli (12). If the fluid is not rapidly cleared, alveolar edema occurs; thus, alveolar fluid clearance (AFC) is critical in preventing ALI/ARDS. Specific ion and water channels are known to participate in AFC. Epithelial $\mathrm{Na}^{+}$channels $(\mathrm{ENaC})$ are ion channels composed of 
Table I. Seawater-induced changes.

\begin{tabular}{|c|c|c|c|c|}
\hline Name & Category & Cell/tissue & Change & (Refs.) \\
\hline B cell lymphoma-2 & Apoptosis-related protein & In vivo & $\uparrow P$ & (14) \\
\hline Cleaved caspase- 3 & Apoptosis-related protein & In vivo, $\mathrm{A} 549, \mathrm{PAT} 2$ & $\uparrow \mathrm{P}$ & $(13,14)$ \\
\hline Cleaved caspase- 8 & Apoptosis-related protein & In vivo, $\mathrm{A} 549, \mathrm{PAT} 2$ & $\uparrow P$ & (13) \\
\hline Fas & Apoptosis-related protein & In vivo, $\mathrm{A} 549, \mathrm{PAT} 2$ & $\uparrow \mathrm{P}$ & (13) \\
\hline FasL & Apoptosis-related protein & In vivo, $\mathrm{A} 549, \mathrm{PAT} 2$ & $\uparrow \mathrm{P}$ & (13) \\
\hline 4E-BP1 & Binding protein & $\mathrm{AEC}$ & $\uparrow \mathrm{Ph}$ & (27) \\
\hline AQP1 & Channel & In vivo, $\mathrm{A} 549, \mathrm{PAT} 2$ & $\uparrow R, P$ & $(21,23)$ \\
\hline AQP5 & Channel & In vivo, $\mathrm{A} 549, \mathrm{PAT} 2$ & $\uparrow R, P$ & $(21,23)$ \\
\hline$\alpha-\mathrm{ENaC}$ & Channel & In vivo & $\downarrow R$ & $(30)$ \\
\hline IL-10 & Cytokine & In vivo & $\uparrow \mathrm{P}$ & $(15-17,28)$ \\
\hline IL-1 $\beta$ & Cytokine & In vivo, $\mathrm{NR} 8383$ & $\uparrow \mathrm{P}$ & $(15-17,27)$ \\
\hline IL-6 & Cytokine & In vivo, $\mathrm{NR} 8383$ & $\uparrow P$ & $(20,27)$ \\
\hline IL-8 & Cytokine & In vivo & $\uparrow \mathrm{P}$ & $(24,25)$ \\
\hline MIF & Cytokine & In vivo & $\uparrow \mathrm{P}$ & (20) \\
\hline TNF- $\alpha$ & Cytokine & In vivo, $\mathrm{NR} 8383$ & $\uparrow \mathrm{P}$ & $(16,17,20,27,28,30)$ \\
\hline MPO & Enzyme & In vivo & $\uparrow A$ & $(20,28,30)$ \\
\hline MYPT-1 & Enzyme & In vivo, A549, RPMVECs & $\uparrow \mathrm{Ph}$ & (31) \\
\hline T-SOD & Enzyme & In vivo & $\downarrow \mathrm{A}$ & $(17)$ \\
\hline VEGF & Growth regulator & In vivo & $\uparrow R, P$ & $(19,22)$ \\
\hline Akt & Kinase & In vivo, $\mathrm{AEC}$ & $\uparrow \mathrm{Ph}$ & $(14,19)$ \\
\hline ATM & Kinase & $\mathrm{AEC}$ & $\uparrow \mathrm{Ph}$ & (19) \\
\hline eIF4E & Kinase & AEC & $\uparrow \mathrm{Ph}$ & (27) \\
\hline JAK1 & Kinase & NR8383 & $\uparrow \mathrm{Ph}$ & $(15)$ \\
\hline JAK2 & Kinase & NR8383 & $\uparrow \mathrm{Ph}$ & $(15)$ \\
\hline p70S6K1 & Kinase & $\mathrm{AEC}$ & $\uparrow \mathrm{Ph}$ & $(27)$ \\
\hline PI3K & Kinase & $\mathrm{AEC}$ & $\uparrow \mathrm{Ph}$ & (19) \\
\hline PKC & Kinase & In vivo, A549 & $\uparrow \mathrm{Ph}$ & $(26)$ \\
\hline ERK $1 / 2$ & MAP kinase & In vivo & $\uparrow \mathrm{Ph}$ & (14) \\
\hline p38 & MAP kinase & AEC & $\uparrow \mathrm{Ph}$ & (19) \\
\hline LC3 & Microtubule associated protein & In vivo & $\uparrow R$ & $(25)$ \\
\hline LC3-II & Microtubule associated protein & In vivo & $\uparrow \mathrm{P}$ & $(25)$ \\
\hline SEMA7A & Neuronal guidance protein & In vivo, $\mathrm{RPMVECs}$ & $\uparrow P$ & (32) \\
\hline MDA & Peroxidation product & In vivo & $\uparrow \mathrm{A}$ & (17) \\
\hline $\mathrm{NE}$ & Protease & In vivo & $\uparrow \mathrm{P}$ & $(28)$ \\
\hline $\mathrm{ER} \beta$ & Receptor & In vivo & $\downarrow \mathrm{P}$ & $(21)$ \\
\hline sVEGFR $_{1}$ & Receptor & In vivo & $\uparrow \mathrm{P}$ & $(22)$ \\
\hline VDR & Receptor & In vivo, A549, RPMVECs & $\uparrow \mathrm{R}, \mathrm{P}$ & $(31)$ \\
\hline S6 ribosomal & Ribosomal protein & $\mathrm{AEC}$ & $\uparrow \mathrm{Ph}$ & (27) \\
\hline SP-A & Secretory protein & In vivo & $\downarrow R$ & $(30)$ \\
\hline GTP-RhoA & Signaling $\mathrm{G}$ protein & In vivo, A549, RPMVECs & $\uparrow P$ & $(31)$ \\
\hline HIF- $1 \alpha$ & Transcription factor & In vivo, $\mathrm{A} 549, \mathrm{AEC}$ & $\uparrow \mathrm{P}$ & $(17,19,27)$ \\
\hline $\mathrm{NF}-\kappa \mathrm{B}$ & Transcription factor & In vivo, $\mathrm{A} 549$ & $\uparrow \mathrm{Ph}, \mathrm{TL}$ & $(17,20,28,31)$ \\
\hline STAT1 & Transcription factor & In vivo, $\mathrm{NR} 8383$ & $\uparrow \mathrm{P}, \mathrm{Ph}$ & (15) \\
\hline $\mathrm{Cx} 43$ & Transmembrane channel & In vivo, A549 & $\uparrow \mathrm{R}, \mathrm{Ph}$ & $(16,26)$ \\
\hline $\mathrm{Cx} 43$ & Transmembrane channel & In vivo & $\downarrow \mathrm{P}$ & (16) \\
\hline $\mathrm{Na}^{+} / \mathrm{K}^{+}$-ATPase & Transporter & In vivo & $\downarrow \mathrm{A}, \mathrm{P}$ & $(29,30)$ \\
\hline
\end{tabular}

4E-BP, eukaryotic translation initiation factor 4E binding protein 1; AQP, aquaporin; $\alpha$-ENaC, epithelial sodium channel subunit alpha; IL, interleukin; MIF, macrophage migration inhibitory factor; TNF- $\alpha$, tumor necrosis factor alpha; MPO, myeloperoxidase; MYPT-1, myosin light-chain phosphatase-1; T-SOD, total superoxide dismutase; VEGF, vascular endothelial growth factor; Akt, protein kinase B; eIF4E, eukaryotic translation initiation factor 4E; JAK, janus kinase; P70S6K1, ribosomal protein S6 kinase beta-1; PI3K, phosphatidylinositol-4,5-bisphosphate 3-kinase; PKC, protein kinase C; ERK, extracellular signal-regulated kinase; LC3, microtubule-associated protein 1 light chain 3 alpha; SEMA7A, semaphorin 7A; MDA, malondialdehyde; NE, neutrophil elastase; ER $\beta$, estrogen receptor beta; sVEGFR 1 , soluble vascular endothelial growth factor receptor 1; VDR, vitamin D receptor; SP-A, secretory protein A; GTP-RhoA, guanosine-5'-triphosphate-ras homolog gene family member A; HIF-1 $\alpha$, hypoxia-inducible factor 1-alpha; NF- $\mathrm{B}$, nuclear factor-kappa B; STAT1, signal transducer and activator of transcription 1; Cx43, connexin 43; A, activity; P, protein abundance; Ph, phosphorylation; A, activity; R, mRNA abundance; TL, translocation; PAT2, primary alveolar type II cells; AEC, alveolar epithelial cells; RPMVECs, rat pulmonary microvascular endothelial cells. 


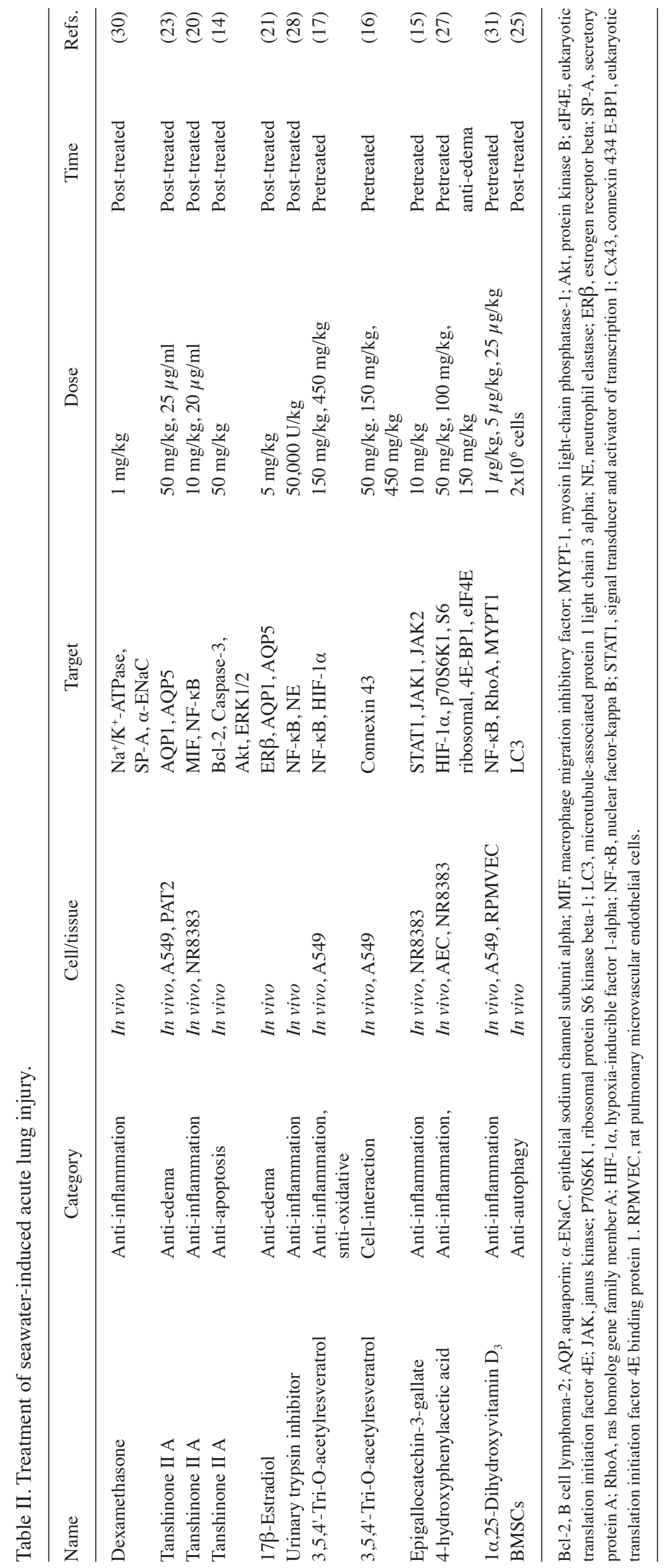


three subunits $(\alpha, \beta$ and $\gamma)(49,50)$. Their key function is $\mathrm{Na}^{+}$ uptake and to aid the removal of excess pulmonary edema fluid from the alveolar space (51). A previous study indicated the importance of AFC by demonstrating that knockout $\alpha$-EnaC mice succumbed within $48 \mathrm{~h}$ following birth, due to their inability to clear alveolar edema fluid (52). $\mathrm{Na}^{+} / \mathrm{K}^{+}$adenosine triphosphatase $\left(\mathrm{Na}^{+} / \mathrm{K}^{+}\right.$-ATPase $)$is a basolateral membrane protein that exchanges sodium and potassium (53). In concert with $\mathrm{ENaC}, \mathrm{Na}^{+} / \mathrm{K}^{+}$-ATPase produces an osmotic gradient that aids in the reabsorption of alveolar fluid (51). As described in previous reports $(54,55)$, impaired $\mathrm{Na}^{+} / \mathrm{K}^{+}$-ATPase may trigger severe lung edema. Aquaporins (AQPs) are a family of integral membrane proteins that contribute to transcellular and trans-epithelial water movement (56). The predominant types exhibited in the lung are AQP1 and AQP5 (57).AQP1 is primarily expressed in the microvascular endothelium, while AQP5 is located in the apical membrane of type II epithelium cells (57). Previous studies $(58,59)$ have indicated that AQP levels are altered in multiple models of lung injury, likely contributing to lung edema formation. For seawater-drowning-induced ALI, the expression levels and activity of $\mathrm{Na}^{+} / \mathrm{K}^{+}$-ATPase in the lung tissue are reduced $(29,30)$. In addition, $\mathrm{ENaC}$ transcription is also decreased (30). In contrast, seawater exposure increases AQP1 and AQP5 expression levels, indicating elevated water permeability of the blood-air barrier $(21,23)$. All these changes promote lung edema.

Inflammation. Development of ALI/ARDS is accompanied by increased inflammatory responses (60). Key ALI/ARDS characteristics induce inflammatory cell adhesion and exudation, which result in the release of large quantities of inflammatory cytokines and chemokines $(15,17,20,32)$. These reaction cascades have an important role in the defense against pathogens (61). However, an abnormal hyperactive inflammatory response may promote and/or aggravate ALI/ARDS. Stimulation by seawater, pathogen invasion and pulmonary edema may all influence neutrophil activation (17), subsequently releasing large quantities of inflammatory cytokines, such as interleukin (IL)-1 $\beta$ (15-17), IL-6 (20,27), IL-8 (25) and tumor necrosis factor- $\alpha(20,25)$. In addition, neutrophilic activation also promotes the release of reactive oxygen species (ROS), in addition to some vasoactive substances (such as hypoxia-inducible factor-1 $\alpha$ and vascular endothelial growth factor), which further aggravate lung injury (17).

Seawater-induced inflammatory cytokine release is associated with multiple pathways, including nuclear factor- $\kappa \mathrm{B}(17)$, hypoxia-inducible factor $-1 \alpha(16,19)$, macrophage migration inhibitory factor (20) and RhoA/Rho kinase signaling (31). Furthermore, seawater inhalation may promote lung injury through activating the Janus kinase/signal transducer and activator of transcription 1 (15) and p38 pathways (19). Inflammatory cytokine release in ALI may promote inflammatory cell activation, creating a vicious cycle, or even a 'cascade effect'.

Oxidative stress. Excessive generation of ROS and oxidative stress are processes that have been indicated in ALI/ARDS, promoting cell injury and apoptosis (62). Inflammatory reactions that occur within airways produce ROS and trigger a redox imbalance in the lungs (63). Indeed, scavenging of ROS significantly attenuates lipopolysaccharide (LPS)-induced lung injury (62). To date, no studies have demonstrated that seawater may promote ROS generation and induce oxidative stress; however, previous results have demonstrated that exposure to seawater induces myeloperoxidase and malondialdehyde activation and decreases total superoxide dismutase activity, indicating that seawater may cause oxidative stress in the lungs (17).

Autophagy and apoptosis. Autophagy is a vital process within the lysosomal degradation pathway. The predominant function of autophagy is the disposal of denatured proteins and damaged organelles (64). Furthermore, autophagy exhibits a homeostatic function at low basal levels and affects multiple critical cellular processes, including cell apoptosis, cell proliferation and immune function (65). Promotion of autophagy is triggered by various stressors, such as hypoxia, oxidative stress and hyperosmosis, which causes abnormal activation of inflammatory reactions or programmed cell death (66). Increasing evidence indicates that autophagy participates in multiple lung diseases (65). Previous studies have indicated that seawater aspiration may activate autophagy $(24,25)$. Indeed, alveolar epithelial cells have been revealed to generate more autophagosomes following treatment with seawater (24) and notable upregulation of the autophagy protein, LC3-II was detected (25). Furthermore, autophagy inhibition by 3 -methyladenine significantly attenuates seawater-inhalation-induced effects by reducing the partial pressure of oxygen, increasing the lung weight coefficient and destroying the alveolar structure (24).

Seawater aspiration is able to induce apoptosis in alveolar epithelial cells $(13,14)$. Apoptosis may be initiated by two pathways: The extrinsic pathway, which is mediated through extracellular ligand binding, including Fas ligand (FasL), to specific receptors on the cell surface; and the intrinsic pathway, which is mediated by the mitochondria (67). Notably, seawater was revealed to activate the extrinsic pathway (13). A previous study indicated that, following seawater inhalation, Fas and FasL levels in the lung were increased to the extent where caspase- 8 and caspase-3 cleavage was induced, which resulted in apoptosis (13). Alternative studies have identified that seawater induced apoptosis by significantly reducing the expression levels of the anti-apoptotic molecule, B-cell lymphoma $2(13,14)$. Activation of Akt, which modulates cell survival and apoptosis, and extracellular signal-regulated kinases 1 and 2 that are involved in the protective action against cell death, was indicated to be triggered immediately following seawater exposure (14).

Hypertonic stimulation. High osmotic pressure is able to inflict cell damage and result in the loss of normal physiological function (68). Intestinal epithelial cells produce inflammatory mediators when exposed to hypertonic fluids, which promotes inflammatory bowel disease (69). Furthermore, stimulation with hypertonic fluids may cause a series of pathological perturbations in various cells in the lung tissue, including lung epithelial and vascular endothelial cell shrinkage, apoptosis, neutrophil chemotaxis, blood-gas barrier damage, as well as infiltration and secretion of inflammatory cytokines (12-15). 
The osmosis-sensitive transcription factor, osmotic response element-binding protein (OREBP) is a member of the Rel transcriptional activators family, which has been previously described (69). OREBP transactivates several genes responsible for cell protection against injury derived from hyperosmosis, such as organic osmolytes (70), heat shock protein 70 (71) and vasopressin-activated urea transporters (72). Although no direct evidence has demonstrated that seawater activates OREBP in ALI/ARDS, it has been reported that multiple OREBP regulators are altered in seawater-drowning-induced ALI/ARDS $(27,68)$.

Increased ROS levels are necessary for OREBP activation; however, these levels may be decreased following ROS reduction by antioxidants (73). Actin cytoskeleton reorganization has been indicated in the activation of OREBP though RhoA/Rho kinase signaling (68). Burg et al (68) reported that seawater-induced changes in p38, ataxia telagiectasia mutated kinase (ATM) and Phosphatidylinositol-4,5-bisphosphate 3-kinases (PI3Ks) regulated OREBP activity. p38 belongs to the mitogen-activated protein kinases family and its inhibition has been revealed to partially reduce hypertonicity-induced activation of OREBP (74). Furthermore, as does ATM, which participates in cell cycle regulation, DNA repair and cell survival. PI3Ks are intracellular lipid kinases may mediate OREBP activity though ATM (68). Therefore, we hypothesize that OREBP, an osmosis sensitive protein, is likely involved in seawater-drowning-induced ALI/ARDS.

Other possible mechanisms. Alternative possible mechanisms related to seawater-inhalation-induced ALI/ARDS include calcium oscillation and intracellular calcium overload. It has been indicated that high salt levels increase the production of pro-inflammatory molecules and potentiate LPS-induced macrophage activation (75); therefore, seawater may elicit similar effects.

\section{Potential treatments and therapeutic targets of seawater-drowning-induced acute lung injury}

Hospital management. Although the pathophysiological and molecular mechanisms of ALI/ARDS are well-acknowledged, no specific and effective treatments are currently available. The main treatment is supportive care, including pulmonary support, to avoid of complications (1).

Potential treatments suggested by animal experiments. Previous studies in rats and rabbits have indicated various treatments (as shown in Table II) that may be effective for seawater inhalation induced ALI/ARDS (Table II; $14-17,20,21,23,25,27,28,30,31)$. These therapeutic strategies predominantly focus on modulating the over-activated immunoreactions, such as dexamethasone, tanshinone II A and 1 $\alpha, 25$-dihydroxyvitamin D3. Additionally, some therapeutic agents are able to alleviate increased edema, such as $17 \beta$-Estradiol and tanshinone II A. However, many of these therapeutic strategies, including the therapeutic agents and their doses, have not been evaluated for safety in humans and the exact effects these may exert requires further investigation.

\section{Conclusions}

To conclude, seawater drowning is a complex injury process that involves pulmonary edema formation, inflammatory response enhancement, oxidative stress, hypertonic stimulation and pathogen invasion. In addition, other possible mechanisms require further exploration; these include the exact roles of OREBP, calcium oscillation and high salt concentrations. For potential treatments, further studies and confirmation are required.

\section{Acknowledgements}

The authors are grateful to Dr Liyan Bo (Department of Respiratory and Critical Care Medicine, Tangdu Hospital, The Fourth Military Medical University, Shaanxi, China) for her support in this review.

\section{References}

1. Layon AJ and Modell JH: Drowning: Update 2009. Anesthesiology 110: 1390-1401, 2009.

2. Global report on drowning: Preventing a leading killer: World Health Organization, 2014.

3. Engel SC: Drowning episodes: Prevention and resuscitation tips J Fam Pract 64: E1-E6, 2015.

4. Gregorakos L, Markou N, Psalida V, Kanakaki M, Alexopoulou A, Sotiriou E, Damianos A and Myrianthefs P: Near-drowning: Clinical course of lung injury in adults. Lung 187: 93-97, 2009.

5. Salomez F and Vincent J: Drowning: A review of epidemiology, pathophysiology, treatment and prevention. Resuscitation 63: 261-268, 2004.

6. Wheeler AP and Bernard GR: Acute lung injury and the acute respiratory distress syndrome: A clinical review. Lancet 369: 1553-1564, 2007

7. Pierrakos C, Karanikolas M, Scolletta S, Karamouzos V and Velissaris D: Acute respiratory distress syndrome: Pathophysiology and therapeutic options. J Clin Med Res 4: 7-16, 2012.

8. Bierens JJLM, Idris A, Bierens J, et al: Recommended Guidelines for Uniform Reporting of Data from Drowning: The Utstein Style. In: Bierens JJLM (ed). Drowning. Springer Berlin Heidelberg, pp757-765, 2014.

9. Vohwinkel CU, Hoegl S and Eltzschig HK: Hypoxia signaling during acute lung injury. J Appl Physiol (1985) 119: 1157-1163, 2015.

10. Tojo K, Nagamine Y, Yazawa T, Mihara T, Baba Y, Ota S, Goto T and Kurahashi K: Atelectasis causes alveolar hypoxia-induced inflammation during uneven mechanical ventilation in rats. Intensive Care Med Exp 3: 56, 2015.

11. Nawa S, Shimizu A, Kino K, Soga H and Shimizu N: Development of an experimental model of an acute respiratory failure by intratracheal sea water infusion: A comparison with a conventional oleic acid induction. Res Exp Med (Berl) 194: 25-33, 1994.

12. Folkesson HG, Kheradmand F and Matthay MA: The effect of salt water on alveolar epithelial barrier function. Am J Respir Crit Care Med 150: 1555-1563, 1994.

13. Han F, Luo Y, Li Y, Liu Z, Xu D, Jin F and Li Z: Seawater induces apoptosis in alveolar epithelial cells via the Fas/FasL-mediated pathway. Respir Physiol Neurobiol 182: 71-80, 2012.

14. Li J, Xu M, Xie X, Fan QX, Mu DG, Zhang Y, Cao FL, Wang YX, Zhao PT, Zhang B, et al: Tanshinone IIA suppresses lung injury and apoptosis and modulates protein kinase B, and extracellular signal-regulated protein kinase pathways in rats challenged with seawater exposure. Clin Exp Pharmacol Physiol 38: 269-277, 2011.

15. Liu W, Dong M, Bo L, Li C, Liu Q, Li Y, Ma L, Xie Y, Fu E, $\mathrm{Mu} \mathrm{D}$, et al: Epigallocatechin-3-gallate ameliorates seawater aspiration-induced acute lung injury via regulating inflammatory cytokines and inhibiting JAK/STAT1 pathway in rats. Mediators Inflamm 2014: 612593,2014.

16. Ma L, Li Y, Zhao Y, Wang Q, Nan Y, Mu D, Li W, Sun R, Jin F and Liu X: 3,5,4'-tri-O-acetylresveratrol ameliorates seawater exposure-induced lung injury by upregulating connexin 43 expression in lung. Mediat Inflamm 2013: 182132, 2013. 
17. Ma L, Zhao Y, Li B, Wang Q, Liu X, Chen X, Nan Y, Liang L, Chang R, Liang L, et al: 3,5,4'-Tri-O-acetylresveratrol attenuates seawater aspiration-induced lung injury by inhibiting activation of nuclear factor-kappa B and hypoxia-inducible factor- $1 \alpha$. Resp Physiol Neurobiol 185: 608-614, 2013.

18. Halmagyi DF: Lung changes and incidence of respiratory arrest in rats after aspiration of sea and fresh water. J Appl Physiol 16: 41-44, 1961.

19. Liu Z, Zhang B, Wang X, Li Y, Xi RG, Han F, Li WP, Fu L, Li Z and Jin F: Hypertonicity contributes to seawater aspiration-induced lung injury: Role of hypoxia-inducible factor $1 \alpha$. Exp Lung Res 41: 301-315, 2015.

20. Zhang Y, Zhang B, Xu DQ, Li WP, Xu M, Li JH, Xie XY, Fan QX, Liu W, Mu DG, et al: Tanshinone IIA attenuates seawater aspiration-induced lung injury by inhibiting macrophage migration inhibitory factor. Biol Pharm Bull 34: 1052-1057, 2011.

21. Fan Q, Zhao P, Li J, Xie X, Xu M, Zhang Y, Mu D, Li W, Sun R, Liu W, et al: $17 \beta$-Estradiol administration attenuates seawater aspiration-induced acute lung injury in rats. Pulm Pharmacol Ther 24: 673-681, 2011

22. Hu X, Duan Y, Li Y and Xue Z: Early responses of VEGF during acute lung injury induced by seawater immersion after open chest trauma. Respiration 79: 490-496, 2010.

23. Li J, Xu M, Fan Q, Xie X, Zhang Y, Mu D, Zhao P, Zhang B, Cao F, Wang Y, et al: Tanshinone IIA ameliorates seawater exposure-induced lung injury by inhibiting aquaporins (AQP) 1 and AQP5 expression in lung. Resp Physiol Neurobi 176: 39-49, 2011.

24. Liu Q, Zhou D, Lin P, Gao X, Pan L and Jin F: Participation of autophagy in acute lung injury induced by seawater. Exp Lung Res 39: 441-452, 2013.

25. Liu Q, Zhou D, Sun L, Ling L, Wu CG, Lin P and Han SP: Bone marrow mesenchymal stem cells ameliorates seawater-exposure-induced acute lung injury by inhibiting autophagy in lung tissue. Pathol Res Int 2014: 104962, 2014

26. Liu T, Li Y, Zhang B, Ma L, Liu W, Li Z and Jin F: The role of phosphorylated Cx43 on PKC mediated Ser368 in lung injury induced by seawater inhalation. Inflammation 38: 1847-1854, 2015.

27. Liu Z, Xi R, Zhang Z, Li W, Liu Y, Jin F and Wang X: 4-hydroxyphenylacetic acid attenuated inflammation and edema via suppressing HIF-1 $\alpha$ in seawater aspiration-induced lung injury in rats. Int J Mol Sci 15: 12861-12884, 2014.

28. Rui M, Duan YY, Zhang XH, Wang HL and Wang DP: Urinary trypsin inhibitor attenuates seawater-induced acute lung injury by influencing the activities of nuclear factor-kB and its related inflammatory mediators. Respiration 83: 335-343, 2012.

29. Xie XY, Zhang B, Li JH, Fan OX, Zhang Y, Mu DG, Li WP, Xu M, Zhao PT, Jin FG and Li ZC: Sodium tanshinone iia sulfonate attenuates seawater aspiration-induced acute pulmonary edema by up-regulating $\mathrm{Na}(+), \mathrm{K}(+)$-ATPase activity. Exp Lung Res 37: 482-491, 2011.

30. Xinmin D, Yunyou D, Chaosheng P, Huasong F, Pingkun Z Jiguang M, Zhiqian X and Qinzhi X: Dexamethasone treatment attenuates early seawater instillation-induced acute lung injury in rabbits. Pharmacol Res 53: 372-379, 2006.

31. Zhang M, Dong M, Liu W, Wang L, Luo Y, Li Z and Jin F: 1a,25-dihydroxyvitamin D3 ameliorates seawater aspiration-induced acute lung injury via NF-kB and RhoA/Rho kinase pathways. PLoS One 9: e104507,2014.

32. Zhang M, Wang L, Dong M, Li Z and Jin F: Endothelial semaphorin $7 \mathrm{~A}$ promotes inflammation in seawater aspiration-induced acute lung injury. Int J Mol Sci 15: 19650-19661, 2014.

33. Mulugeta S, Nureki SI and Beers MF: Lost after translation: Insights from surfactant for understanding the role of alveolar epithelial dysfunction and cell quality control in fibrotic lung disease. Am J Physiol Lung Cell Mol Physiol 309: L507-L525, 2015.

34. Goto H, Mitsuhashi A and Nishioka Y: Role of surfactant protein A in non-infectious lung diseases. J Med Invest 61: 1-6, 2014

35. Puntorieri V, Hiansen JQ, McCaig LA, Yao LJ, Veldhuizen RA and Lewis JF: The effects of exogenous surfactant administration on ventilation-induced inflammation in mouse models of lung injury. BMC Pulm Med 13: 67, 2013.

36. Ou CY, Chen CZ, Hsiue TR, Lin SH and Wang JY: Genetic variants of pulmonary SP-D predict disease outcome of COPD in a Chinese population. Respirology 20: 296-303, 2015.

37. Liu W, Ju CR, Chen RC and Liu ZG: Role of serum and induced sputum surfactant protein $\mathrm{D}$ in predicting the response to treatment in chronic obstructive pulmonary disease. Exp Ther Med 8: $1313-1317,2014$
38. Morissette MC, Shen P, Thayaparan D and Stämpfli MR: Disruption of pulmonary lipid homeostasis drives cigarette smoke-induced lung inflammation in mice. Eur Respir J 46: 1451-1460, 2015

39. Olmeda B, Garcia-Álvarez B, Gómez MJ, Martínez-Calle M, Cruz A and Pérez-Gil J: A model for the structure and mechanism of action of pulmonary surfactant protein B. FASEB J 29: 4236-4247, 2015

40. Bloomfield GL, Sweeney LB, Fisher BJ, Blocher CR, Sholley MM, Sugerman HJ and Fowler AA III: Delayed administration of inhaled nitric oxide preserves alveolar-capillary membrane integrity in porcine gram-negative sepsis. Arch Surg 132: 65-75, 1997.

41. Chen J, Shao Y, Xu G, Lim C, Li J, Xu D and Shen J: Bone marrow-derived mesenchymal stem cells attenuate phosgene-induced acute lung injury in rats. Inhal Toxicol 27: 254-261, 2015.

42. Kondrikov D, Gross C, Black SM and Su Y: Novel peptide for attenuation of hyperoxia-induced disruption of lung endothelial barrier and pulmonary edema via modulating peroxynitrite formation. J Biol Chem 289: 33355-33363, 2014.

43. Aeffner F, Bolon B and Davis IC: Mouse models of acute respiratory distress syndrome: A review of analytical approaches, pathologic features, and common measurements. Toxicol Pathol 43: 1074-1092, 2015.

44. Yang B, Li XP, Ni YF, Du HY, Wang R, Li MJ, Wang WC, Li MM, Wang XH, Li L, et al: Protective effect of isorhamnetin on lipopolysaccharide-induced acute lung injury in mice. Inflammation 39: 129-137, 2016.

45. Amano M, Chihara K, Kimura K, Fukata Y, Nakamura N, Matsuura Y and Kaibuchi K: Formation of actin stress fibers and focal adhesions enhanced by Rho-kinase. Science 275: 1308-1311, 1997.

46. Amano M, Ito M, Kimura K, Fukata Y, Chihara K, Nakano T, Matsuura Y and Kaibuchi K: Phosphorylation and activation of myosin by Rho-associated kinase (Rho-kinase). J Biol Chem 271: 20246-20249, 1996.

47. Takai Y, Sasaki T and Matozaki T: Small GTP-binding proteins. Physiol Rev 81: 153-208, 2001.

48. Laird DW: Connexin phosphorylation as a regulatory event linked to gap junction internalization and degradation. Biochim Biophys Acta 1711: 172-182, 2005.

49. Althaus M, Clauss WG and Fronius M: Amiloride-sensitive sodium channels and pulmonary edema. Pulm Med 2011: 830320, 2011

50. Canessa CM, Schild L, Buell G, Thorens B, Gautschi I, Horisberger JD and Rossier BC: Amiloride-sensitive epithelial $\mathrm{Na}^{+}$channel is made of three homologous subunits. Nature 367: 463-467, 1994

51. Matthay MA, Folkesson HG and Clerici C: Lung epithelial fluid transport and the resolution of pulmonary edema. Physiol Rev 82: 569-600, 2002.

52. Matalon S, Lazrak A, Jain L and Eaton DC: Invited review: Biophysical properties of sodium channels in lung alveolar epithelial cells. J Appl Physiol (1985) 93: 1852-1859, 2002.

53. Lingrel JB and Kuntzweiler T: $\mathrm{Na}^{+}, \mathrm{K}(+)$-ATPase. J Biol Chem 269: 19659-19662, 1994.

54. Mutlu GM, Machado-Aranda D, Norton JE, Bellmeyer A, Urich D, Zhou R and Dean DA: Electroporation-mediated gene transfer of the $\mathrm{Na}^{+}, \mathrm{K}^{+}$-ATPase rescues endotoxin-induced lung injury. Am J Respir Crit Care Med 176: 582-590, 2007.

55. Vadasz I, Morty RE, Kohstall MG, Olschewski A, Grimminger F, Seeger W and Ghofrani HA: Oleic acid inhibits alveolar fluid reabsorption: A role in acute respiratory distress syndrome? Am J Respir Crit Care Med 171: 469-479, 2005.

56. Agre P, King LS, Yasui M, Guggino WB, Ottersen OP, Fujiyoshi Y, Engel A and Nielsen S: Aquaporin water channels-from atomic structure to clinical medicine. J Physiol 542: 3-16, 2002.

57. Verkman AS: Role of aquaporins in lung liquid physiology. Respir Physiol Neurobiol 159: 324-330, 2007.

58. Lin F, Pan LH, Ruan L, Qian W, Liang R, Ge WY and Huang B: Differential expression of HIF-1a, AQP-1, and VEGF under acute hypoxic conditions in the non-ventilated lung of a one-lung ventilation rat model. Life Sci 124: 50-55, 2015.

59. Gao Z, Xu J, Sun D, Zhang R, Liang R, Wang L and Fan R: Traditional Chinese medicine, Qing Ying Tang, ameliorates the severity of acute lung injury induced by severe acute pancreatitis in rats via the upregulation of aquaporin-1. Exp Ther Med 8: 1819-1824, 2014. 
60. Frank EA, Birch ME and Yadav JS: MyD88 mediates in vivo effector functions of alveolar macrophages in acute lung inflammatory responses to carbon nanotube exposure. Toxicol Appl Pharmacol 288: 322-329, 2015.

61. Ramphal R, Balloy V, Jyot J, Verma A, Si-Tahar M and Chignard M: Control of Pseudomonas aeruginosa in the lung requires the recognition of either lipopolysaccharide or flagellin. J Immunol 181: 586-592, 2008.

62. Chen XJ, Zhang B, Hou SJ, Shi Y, Xu DQ, Wang YX, Liu ML, Dong HY, Sun RH, Bao ND, et al: Osthole improves acute lung injury in mice by up-regulating Nrf-2/thioredoxin 1. Respir Physiol Neurobiol 188: 214-222, 2013.

63. Mannam P, Srivastava A, Sugunaraj JP, Lee PJ and Sauler M: Oxidants in acute and chronic lung disease. J Blood Lymph 4: 1000128,2014

64. Ryter SW, Nakahira K, Haspel JA and Choi AM: Autophagy in pulmonary diseases. Annu Rev Physiol 74: 377-401, 2012.

65. Ryter SW and Choi AM: Autophagy in the lung. Proc Am Thorac Soc 7: 13-21, 2010

66. Zhang J, Wang JS, Zheng ZK, Tang J, Fan K, Guo Ha and Wang JJ: Participation of autophagy in lung ischemia-reperfusion injury in vivo. J Surg Res 182: e79-e87, 2013.

67. Mahata B, Biswas S, Rayman P, Chahlavi A, Ko J, Bhattacharjee A, Li YT, Li Y, Das T, Sa G, et al: GBM derived gangliosides induce $\mathrm{T}$ cell apoptosis through activation of the caspase cascade involving both the extrinsic and the intrinsic pathway. Plos One 10: e0134425, 2015.

68. Burg MB, Ferraris JD and Dmitrieva NI: Cellular response to hyperosmotic stresses. Physiol Rev 87: 1441-1474, 2007.
69. Yan Y, Laroui H, Ingersoll SA, Ayyadurai S, Charania M, Yang S, Dalmasso G, Obertone TS, Nguyen H, Sitaraman SV and Merlin D: Overexpression of Ste20-related proline/alanine-rich kinase exacerbates experimental colitis in mice. J Immunol 187: 1496-1505, 2011

70. Gallazzini M, Ferraris JD, Kunin M, Morris RG and Burg MB: Neuropathy target esterase catalyzes osmoprotective renal synthesis of glycerophosphocholine in response to high $\mathrm{NaCl}$. Proc Natl Acad Sci USA 103: 15260-15265, 2006.

71. Woo SK, Lee SD, Na KY, Park WK and Kwon HM: TonEBP/NFAT5 stimulates transcription of HSP70 in response to hypertonicity. Mol Cell Biol 22: 5753-5760, 2002.

72. Nakayama Y, Peng T, Sands JM and Bagnasco SM: The TonE/TonEBP pathway mediates tonicity-responsive regulation of UT-A urea transporter expression. J Biol Chem 275: 38275-38280, 2000.

73. Zhou X, Ferraris JD and Burg MB: Mitochondrial reactive oxygen species contribute to high $\mathrm{NaCl}$-induced activation of the transcription factor TonEBP/OREBP. Am J Physiol Renal Physiol 290: F1169-F1176, 2006.

74. Ko BC, Lam AK, Kapus A, Fan L, Chung SK and Chung SS: Fyn and p38 signaling are both required for maximal hypertonic activation of the osmotic response element-binding protein/tonicity-responsive enhancer-binding protein (OREBP/TonEBP). J Biol Chem 277: 46085-46092, 2002.

75. Zhang WC, Zheng XJ, Du LJ, Sun JY, Shen ZX, Shi C, Sun S, Zhang Z, Chen XQ, Qin M, et al: High salt primes a specific activation state of macrophages, M(Na). Cell Res 25: 893-910, 2015. 